\title{
Resveratrol ameliorates low shear stress-induced oxidative stress by suppressing ERK/eNOS-Thr495 in endothelial cells
}

\author{
ZHIMEI WANG, JUNXIA ZHANG, BING LI, XIAOFEI GAO, YANRONG LIU, \\ WENXING MAO and SHAO-LIANG CHEN \\ Department of Cardiology, Nanjing First Hospital, Nanjing Medical University, \\ Nanjing, Jiangsu 210006, P.R. China
}

Received October 9, 2013; Accepted May 9, 2014

DOI: $10.3892 / \mathrm{mmr} .2014 .2390$

\begin{abstract}
Fluid shear stress has been revealed to differentially regulate endothelial nitric oxide synthase (eNOS) distribution in vessels. eNOS, a key enzyme in controlling nitric oxide (NO) release, has a crucial role in mediating oxidative stress, and resveratrol (RSV)-mediated eNOS also attenuates oxidative damage and suppresses endothelial dysfunction. To observe the protective effect of RSV on low shear stress (LSS)-induced oxidative damage and the potential mechanisms involved, a parallel-plate flow chamber, which imposed a low level of stress of 2 dynes $/ \mathrm{cm}^{2}$ to cells, was employed. Reactive oxygen species (ROS), NO and apoptotic cells were examined in LSS-treated endothelial cells (ECs) with or without RSV. Western blot analysis was used to examine LSS-regulated eNOS-Ser1177, Thr495 and Ser633, which were tightly associated with NO release. To further determine the underlying signaling pathways involved, extracellular signal-regulated kinase (ERK), a possible upstream target of eNOS-Thr495, was investigated, followed by examination of eNOS-Thr495 in ERK-inhibited cells. Additionally, eNOS mRNA expression levels were analyzed in cells challenged with LSS. The results revealed that RSV markedly decreased LSS-induced oxidative damage in ECs. Furthermore, eNOS-Ser1177 and Thr495 as well as phospho-ERK were time-dependently activated by LSS. The ERK inhibitor deactivated eNOS-Thr495, which was accompanied by increased intracellular superoxide dismutase (SOD) levels. Of note, the activation effect of LSS on ERK/eNOS was markedly eliminated by RSV. In conclusion, RSV exerts antioxidant effects by suppressing LSS-activated ERK/eNOS and may provide a potential therapeutic target for atherosclerosis.
\end{abstract}

Correspondence to: Professor Shao-Liang Chen, Department of Cardiology, Nanjing First Hospital, Nanjing Medical University, 68 Changle Road, Nanjing, Jiangsu 210006, P.R. China

E-mail: chmengx@126.com

Key words: resveratrol, shear stress, endothelial cells, oxidative stress, nitric oxide

\section{Introduction}

Atherosclerosis, a state of excessive oxidative stress, is characterized by endothelial dysfunction. Atherosclerotic plaques, which have a crucial role in the occurrence and progression of atherosclerosis, have been reported to preferentially settle at the branches, bifurcations or inner curvatures of the artery, where shear stress is low $(1,2)$. Shear stress, a dragging force generated by blood flow, exerts a variety of effects on endothelial function and contributes to the focal location of atherosclerotic lesions (3). Several studies have identified that the expression of endothelial nitric oxide synthase (eNOS) protein is markedly increased in high shear stress regions and decreased by low shear stress (LSS) (4-6). eNOS, a critical enzyme that converts L-arginine to L-citrulline and nitric oxide (NO), is important in maintaining endothelial function (7), as the release of $\mathrm{NO}$ affects the formation, progression and heterogeneity of atherosclerotic plaques $(8,9)$. The regulation of eNOS activity involves phosphorylation at multiple serine and threonine residues. The phosphorylation of eNOS-Ser1177 near the carboxy-terminus is required for eNOS activation, and eNOS-Ser633, located in the flavin mononucleotide binding domain, also activates eNOS and is pivotal for the maintenance of NO biosynthesis. eNOS activation is inhibited by eNOS-Thr495, which interferes with the integration of calmodulin at the eNOS calmodulin-binding domain $(6,8,10)$.

Atherogenic events, including decreased NO bioactivity and enhanced formation of reactive oxygen species (ROS), are tightly associated with atherosclerosis formation. The accumulation of ROS promotes apoptosis, leading to high endothelial cell (EC) turnover, which promotes hotspots of increased endothelial permeability and results in a preferred cellular deposition to oxidized low density lipoproteins (11). Additionally, oxidative stress contributes to vascular injury and variously pathological processes, including atherosclerosis and thrombosis (12).

Resveratrol (RSV), a natural polyphenol in grapes, pomegranates and peanuts, first attracted the attention of investigators when it was correlated to the various biological activities of red wine. RSV possesses diverse antiatherogenic effects, including inhibiting low-density lipoprotein oxidation, ameliorating cell apoptosis, disrupting platelet aggregation and suppressing inflammatory factor secretion (13-15). Furthermore, studies 
have reported that RSV has promising neuroprotective and mitochondrial-improving functions, may attenuate aging and prevent the onset of numerous chronic diseases (16-18). In addition, RSV has the ability to scavenge free oxygen radicals and has cytoprotective effects (18-20). Several studies indicated that RSV is able to enhance NO biosynthesis, which may be associated with the extracellular signal-regulated kinase (ERK) signaling pathway (21-23).

The regulatory mechanisms of RSV in the occurrence of endothelial dysfunction induced by LSS have largely remained elusive. In the present study, it was demonstrated that ERK/eNOS is activated by LSS and that RSV inhibits LSS-induced oxidative stress by suppressing ERK/eNOS signaling. Therefore, RSV, as a potential regulator of eNOS, has a protective role in LSS-induced development of atherosclerosis lesion.

\section{Materials and methods}

Cell culture.Human umbilical vein endothelial cells (HUVECs; Type Culture Collection of the Chinese Academy of Sciences, Shanghai, China) were cultured at $37^{\circ} \mathrm{C}$ in a humidified incubator with $5 \% \mathrm{CO}_{2}$ and maintained in RPMI-1640 culture medium supplemented with $10 \%$ fetal bovine serum (FBS; HyClone, Logan, UT, USA). When grown to $90 \%$ confluence, cells were trypsinized, harvested, resuspended and seeded onto a $0.1 \%$ gelatin-coated glass. Following adherence, monolayer cells grown on the glass were used for the flow experiments.

Shear stress study. A parallel-plate flow chamber (Shanghai Medical Instrumentation College, Shanghai, China) that exerts a continuous flow was established by sandwiching a silicon gasket between two stainless steel plates with a cover slip sink in the base plate. The chamber and all parts of the circuit tubes were sterilized prior to the placement of the glass (50x30 mm) with monolayer cells into the flow chamber. The parameter settings were as follows: Flow chamber height, $0.56 \mathrm{~mm}$ and pump rate, 60 times $/ \mathrm{min}$. The value of shear stress was adjusted by modulating the flow of after-loading and was automatically calculated using the information from the pressure transducers. The shear stress in our flow study was 2 dynes $/ \mathrm{cm}^{2}$.

MTT assay. An MTT assay was employed to determine cell viability. Following exposure to LSS for different durations or treatment with different concentrations of RSV (Sigma, St. Louis, MO, USA) for $1 \mathrm{~h}$, cells were incubated in MTT $(0.5 \mathrm{mg} / \mathrm{ml}$; Sigma) for $3 \mathrm{~h}$. Following that, cells were washed twice with ice-cold PBS and DMSO was added to solubilize the converted dye. Finally, DMSO with formazan was collected and the absorbance was measured at $490 \mathrm{~nm}$ by a spectrophotometer (NanoDrop 2000; Thermo Scientific, Waltham, MA, USA).

Detection of ROS and NO. MitoSOX ${ }^{\mathrm{TM}}$ is a live-cell permeant prober and selectively detects superoxide in mitochondria. In the mitochondria, MitoSOX is oxidized by superoxide and acquires red fluorescent properties. 4,5-diaminofluorescein diacetate (DAF-2DA), a membrane-permeable probe, enters cells and is converted into a product with green fluores- cence in the presence of NO. In the present study, the glass with a monolayer of confluent cells was placed into the flow chamber and the cells were subjected to LSS of 2 dynes $/ \mathrm{cm}^{2}$ for 60 min with serum-free RPMI-1640 culture medium as the perfusate. For the RSV group, cells were pretreated with RSV for $30 \mathrm{~min}$ and subjected to a stress of 2 dynes $/ \mathrm{cm}^{2}$ with serum-free RPMI-1640 medium containing the same concentration of RSV as the cycle fluid. To measure ROS and NO, cells following the flow study were gently washed twice with ice-cold phosphate-buffered saline (PBS) and incubated with either MitoSOX (5 $\mu \mathrm{mol} / 1$; Invitrogen Life Technologies, Carlsbad, CA, USA) or DAF-2DA ( $5 \mu \mathrm{mol} / 1$; Beyotime Institute of Biotechnology, Jiangsu, China) at $37^{\circ} \mathrm{C}$ for $20 \mathrm{~min}$ under the exclusion of light. Following this, the cells were washed twice with ice-cold PBS and their nuclei were labeled with DAPI. The images were obtained using a fluorescence microscope (Olympus IX71; Olympus, Tokyo, Japan).

Measuring apoptotic cells. The terminal deoxynucleotidyl transferase dUTP nick end labeling (TUNEL) reaction preferentially labels DNA strand breaks which are produced during apoptosis. In the present study, the cells exposed to LSS with RSV treated or not, were fixed in $4 \%$ paraformaldehyde and permeabilized in $0.1 \%$ sodium citrate containing $0.1 \%$ Triton X-100. Following blockade with $3 \% \mathrm{H}_{2} \mathrm{O}_{2}$ for $10 \mathrm{~min}$, the cells were incubated with TUNEL (Roche Diagnostics, Indianapolis, IN, USA) reaction mixture at room temperature for $1.5 \mathrm{~h}$. TUNEL-positive cells which glowed green fluorescence were captured by a fluorescence microscope.

Western blot analysis. Monolayer cells on a glass were exposed to LSS for different durations with serum free RPMI-1640 medium as the cycle fluid. In the inhibition group, cells were incubated with PD98059 (50 $\mu \mathrm{mol} / \mathrm{ml}$; Sigma), an inhibitor of ERK, prior to flow exposure. Following the flow experiment, cells were lysed in a cocktail of radioimmunoprecipitation assay buffer (50 mM Tris- $\mathrm{HCl}$ pH 7.5, $75 \mathrm{mM} \mathrm{NaCl}$, $15 \mathrm{mM}$ EGTA, $1 \mathrm{mM}$ dithiothreitol, 0.1\% Tween-20, $60 \mathrm{mM}$ glycerophosphate, $1 \mathrm{mM} \mathrm{NaF}, 0.2 \mathrm{mM}$ sodium orthovanadate and $2 \mathrm{mM}$ sodium pyrophosphate; Beyotime Institute of Biotechnology), proteinase inhibitor (Sigma) and phosphatase inhibitor (Roche Diagnostics), and lysates were clarified by centrifugation at $12,000 \mathrm{x}$ g for $15 \mathrm{~min}$ at $4^{\circ} \mathrm{C}$. Protein concentrations were quantified by a bicinchoninic acid protein assay according to the manufacturer's instructions (KeyGen Biotech. Co. Ltd., Nanjing, China). In total, $60 \mu \mathrm{g}$ protein was separated by $10 \%$ SDS-PAGE and transferred to a polyvinylidene fluoride membrane which was then incubated overnight at $4^{\circ} \mathrm{C}$ with primary antibody. Following this, the membrane was washed and incubated with horseradish peroxidase-conjugated secondary antibody for $1 \mathrm{~h}$ at room temperature. Following a second wash, the membranes were developed using enhanced chemiluminescence substrates and the band intensities were analyzed using Image $\mathbf{J}$ software (National Institutes of Health, Bethesda, MD, USA). Goat anti-rabbit IgG-horseradish peroxidase-coupled secondary antibody $(1: 2,000)$ and primary rabbit antibodies against phospho-eNOS-Thr495 (1:1,000), phospho-eNOS-Ser1177 (1:1,000), total eNOS $(1: 1,000)$, ERK 1/2 (1:1,000), phospho-ERK 1/2 (1:2,000) and GAPDH $(1: 2,000)$ were purchased from Cell Signaling Technology, Inc. 
(Beverly, MA, USA). Phospho-eNOS-Ser633 rabbit antibody (1:500) was obtained from Abcam (Cambridge, MA, USA).

Determination of superoxide dismutase (SOD) activity. The SOD assay is based on the formation of formazan from the reaction of 2-(4-iodophenyl)-3-(4-nitrophenyl)-5-(2,4-disulfo phenyl)-2H-tetrazolium, monosodium salt and a superoxide radical produced intracellularly, which is assayed at $450 \mathrm{~nm}$. Cells exposed to LSS for 60 min with or without ERK inhibition were lysed, scraped and centrifuged at $12,000 \mathrm{x} \mathrm{g}$ for $15 \mathrm{~min}$ at $4^{\circ} \mathrm{C}$. The supernatant was collected and the SOD activity was analyzed using a Total SOD assay kit with WST-1 according to the manufacturer's instructions (Beyotime Institute of Biotechnology).

Measurement of intracellular lactate dehydrogenase ( $L D H)$. $\mathrm{LDH}$, an enzyme that leaks from membrane-disturbed cells, was analyzed to confirm the protection of RSV on LSS-treated cells. Cells subjected to LSS for $60 \mathrm{~min}$ with or without RSV treatment were lysed, scraped and centrifuged. Subsequently, the supernatant was collected and the concentration was determined. Intracellular LDH was measured using an LDH assay kit according to the manufacturer's instructions (Nanjing Jiancheng Bioengineering Institute, Nanjing, China).

Quantitative polymerase chain reaction ( $q P C R)$. Total RNA was reverse transcribed into cDNA using a PrimeScript ${ }^{\circledR}$ 1st strand cDNA Synthesis kit (Takara, Dalian, China) and amplification was conducted by $\mathrm{SYBR}^{\circledR}$ Premix Ex $\mathrm{Taq}^{\mathrm{TM}}$ (Takara). eNOS mRNA expression levels were analyzed by qPCR (Applied Biosystems, Foster City, CA, USA) in three independent experiments. The conditions were as follows: $38^{\circ} \mathrm{C}$ for $2 \mathrm{~min}$ and amplification at $95^{\circ} \mathrm{C}$ for $10 \mathrm{~min}$, followed by 40 cycles at $95^{\circ} \mathrm{C}$ for $15 \mathrm{sec}$ and annealed at $60^{\circ} \mathrm{C}$ for $1 \mathrm{~min}$. The primers targeting human eNOS were 5'-TGTTTCTGTCTGCATGG-3' (forward) and 5'-TGGCTGGTAGCGGAAGG-3' (reverse), and those targeting human GAPDH were 5'-GACCTGACCTGCCGTCTA-3' (forward) and 5'-AGGAGTGGGTGTCGCTGT-3' (reverse) (Invitrogen Life Technologies). The relative expression levels of genes were calculated according to the $2^{-\Delta \Delta C T}$ method and eNOS mRNA was normalized to the expression of GAPDH.

Statistical analysis. Values are expressed as the mean \pm standard deviation. Student's t-test was used to analyze data between two groups and analysis of variance was utilized to compare data from more than two groups. $\mathrm{P}<0.05$ was considered to indicate a statistically significant difference. Statistical analyses were performed by SPSS, version 16.0 (SPSS, Inc. Chicago, IL, USA).

\section{Results}

$R S V$ restores LSS-mediated decreases in cell viability. Cell viability was evaluated by an MTT assay. In order to determine the proper concentration, RSV at concentrations of $0-100 \mu \mathrm{mol} / 1$ was used. RSV at $100 \mu \mathrm{mol} / 1$ exhibited a cytotoxic effect (Fig. 1A). Cell viability was markedly decreased in

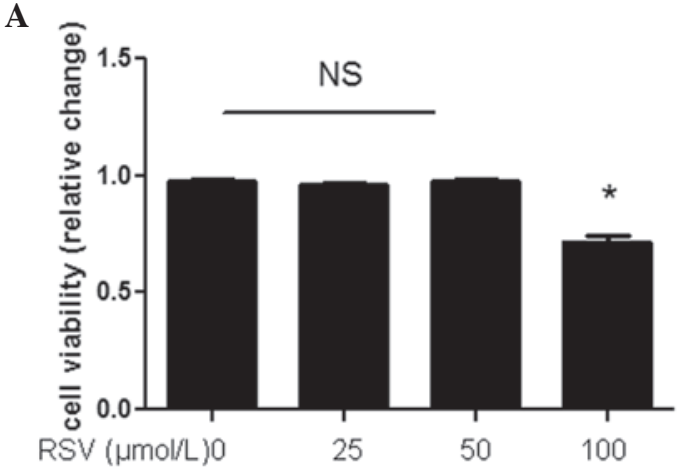

B

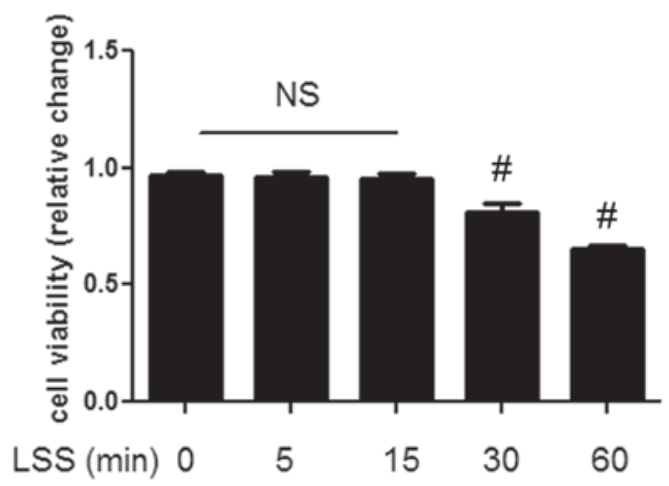

C

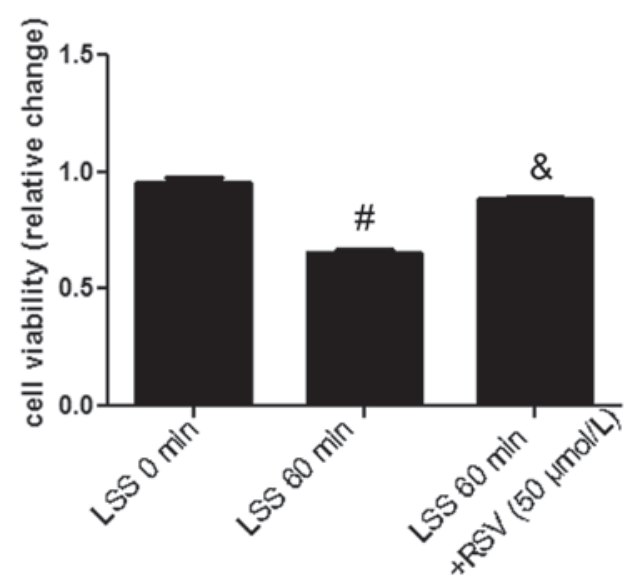

Figure 1. Cell viability was determined by an MTT assay. (A) Endothelial cells were treated with different concentrations of RSV. At the concentration of $100 \mu \mathrm{mol} / 1$, RSV exhibited cytotoxicity. (B) LSS decreased cell viability in a time-dependent manner. (C) RSV at $50 \mu \mathrm{mol} / 1$ restored LSS-induced cell damage. ${ }^{*} \mathrm{P}<0.05$ vs. RSV $0 \mu \mathrm{mol} / 1 ;{ }^{*} \mathrm{P}<0.05$ vs. LSS $0 \mathrm{~min} ;{ }^{\circledR} \mathrm{P}<0.05$ vs. LSS 60 min without RSV; n=3/group. RSV, resveratrol; LSS, low shear stress; NS, not significant.

the presence of LSS (Fig. 1B), which was restored by $50 \mu \mathrm{mol} / 1$ RSV (Fig. 1C). Considering these data, RSV at a concentration of $50 \mu \mathrm{mol} / 1$ was used in the present study.

RSV attenuates LSS-induced oxidative stress. First, the effects of LSS and RSV on ROS production in ECs were investigated. Following $60 \mathrm{~min}$ of exposure to LSS, the ROS levels exhibited a marked increase, which was attenuated by RSV treatment. However, RSV did not affect the levels of ROS in the static cultured cells (Fig. 2A). Due to the fact that excess ROS would impair eNOS function, intracellular NO levels were measured with DAF-2DA. NO levels decreased 
A
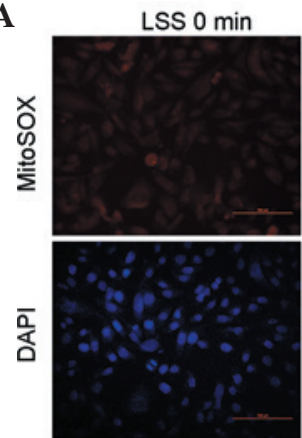
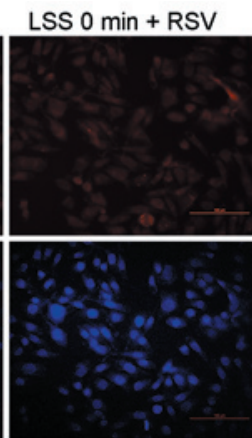

LSS $60 \mathrm{~min}$

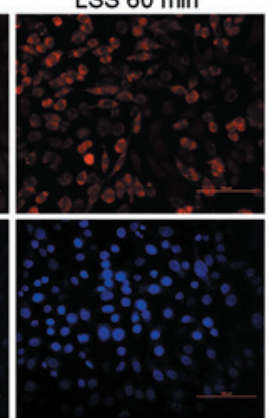

LSS $60 \mathrm{~min}+\mathrm{RSV}$

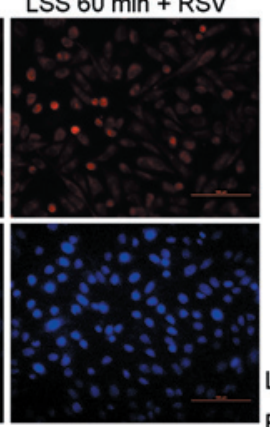

B

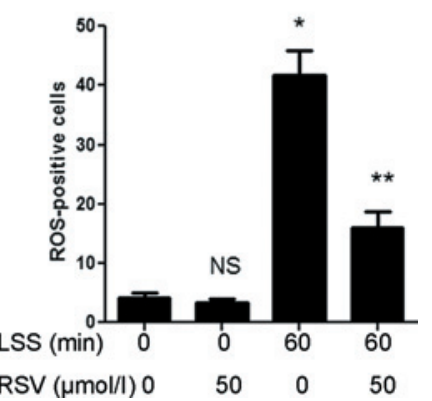

Figure 2. Effects of LSS and RSV on oxidative stress. ROS were visualized using MitoSOX ${ }^{\mathrm{TM}}$. (A) Representative images of ROS-positive cells in red and total nuclei were stained with DAPI (magnification, $\mathrm{x} 200$ ). (B) Bar chart quantifying ROS-positive cells. * P<0.05 vs. LSS 0 min and ${ }^{* *} \mathrm{P}<0.05$ vs. LSS 60 min; $\mathrm{n}=5 /$ group. Scale bar, $100 \mu \mathrm{m}$. LSS, low shear stress; RSV, resveratrol; ROS, reactive oxygen species; NS, not significant.

A

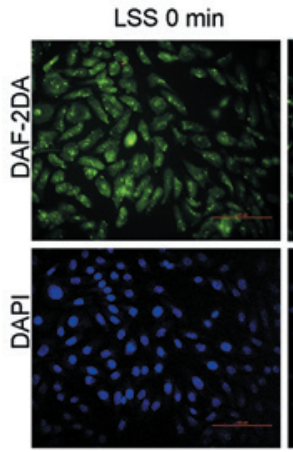

LSS 0 min + RSV

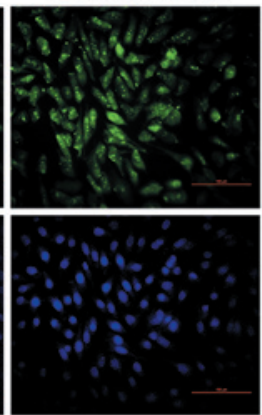

LSS $60 \mathrm{~min}$

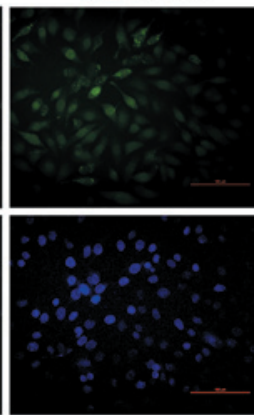

LSS 60 min +RSV

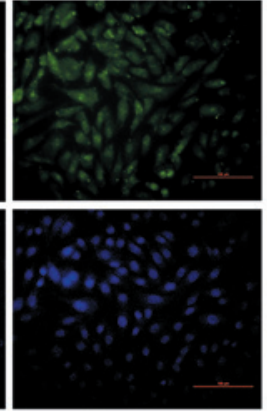

B

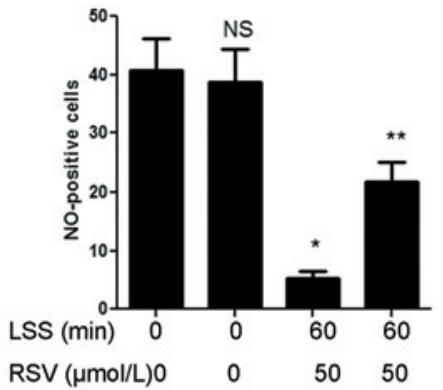

Figure 3. Effects of LSS and RSV on NO release. The NO levels were evaluated by DAF-2DA. (A) Representative images of green NO-positive cells and total nuclei were stainied with DAPI (magnification, x200). (B) Bar chart quantifying NO-positive cells. ${ }^{*} \mathrm{P}<0.05$ vs. LSS 0 min and ${ }^{* *} \mathrm{P}<0.05$ vs. LSS 60 min; $\mathrm{n}=5$ /group. Scale bar, $100 \mu \mathrm{m}$. LSS, low shear stress; RSV, resveratrol; NO, nitric oxide; NS, not significant; DAF-2DA, 4,5-diaminofluorescein diacetate.

A

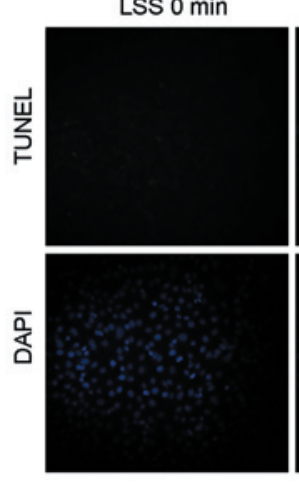

LSS 0 min + RSV

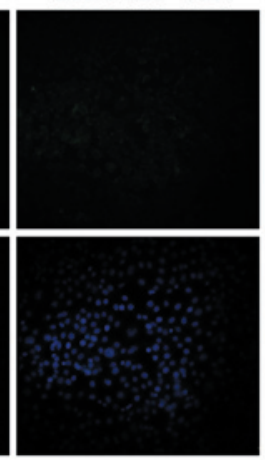

LSS $60 \mathrm{~min}$

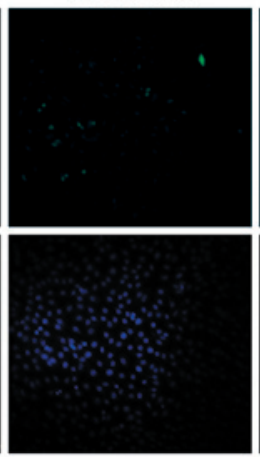

LSS $60 \mathrm{~min}+\mathrm{RSV}$

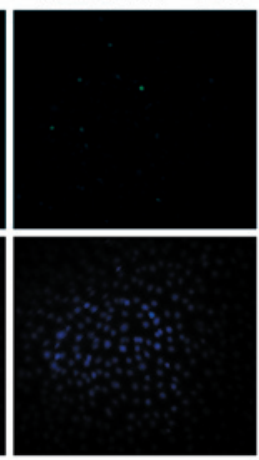

B

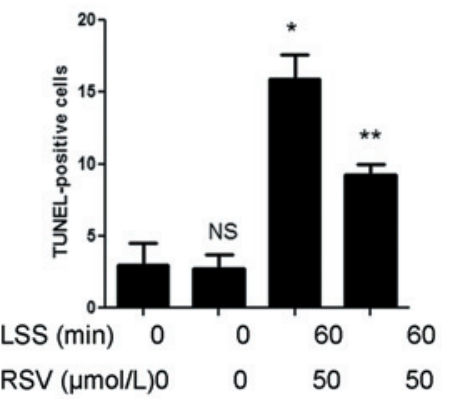

Figure 4. Effects of LSS and RSV on cell apoptosis. (A) Representative images of TUNEL-positive cells and total nuclei stained with DAPI (magnification, x200). (B) Bar chart revealing quantitative data of TUNEL-positive cells. " $\mathrm{P}<0.05$ vs. LSS 0 min and ** P $<0.05$ vs. LSS 60 min, $\mathrm{n}=5 /$ group. Scale bar, $100 \mu \mathrm{m}$. LSS, low shear stress; RSV, resveratrol; NS, not significant; TUNEL, terminal deoxynucleotidyl transferase dUTP nick end labeling.

following 60 min of LSS compared with the LSS 0 min group and were significantly increased following RSV treatment. Similar to the ROS levels, the levels of NO were not affected by RSV under static conditions (Fig. 3A). Considering that oxidative stress-induced apoptosis indicates endothelial dysfunction, TUNEL staining was used to detect apoptotic cells. Cellular apoptosis was markedly increased by LSS, which was attenuated with RSV treatment (Fig. 4A). These data suggested that RSV exhibited antioxidant effects against LSS-induced oxidative damage.
LSS activates eNOS-The495 and-Ser1177 in a time-dependent manner. To determine whether LSS activates eNOS at Thr495, Ser1177 and Ser633, which are closely associated with eNOS activity, multi-phosphorylation sites of eNOS were examined after the cells were subjected to LSS for different durations $(0,5$, 15, 30 and $60 \mathrm{~min}$ ). LSS significantly activated eNOS at Thr495 and Ser1177 sites in a time-dependent manner, while it had no effect eNOS-Ser633 (Fig. 5A). However, acute nutritional deprivation may affect protein phosphorylation. For instance, the substitution of culture medium supplemented with $10 \%$ 


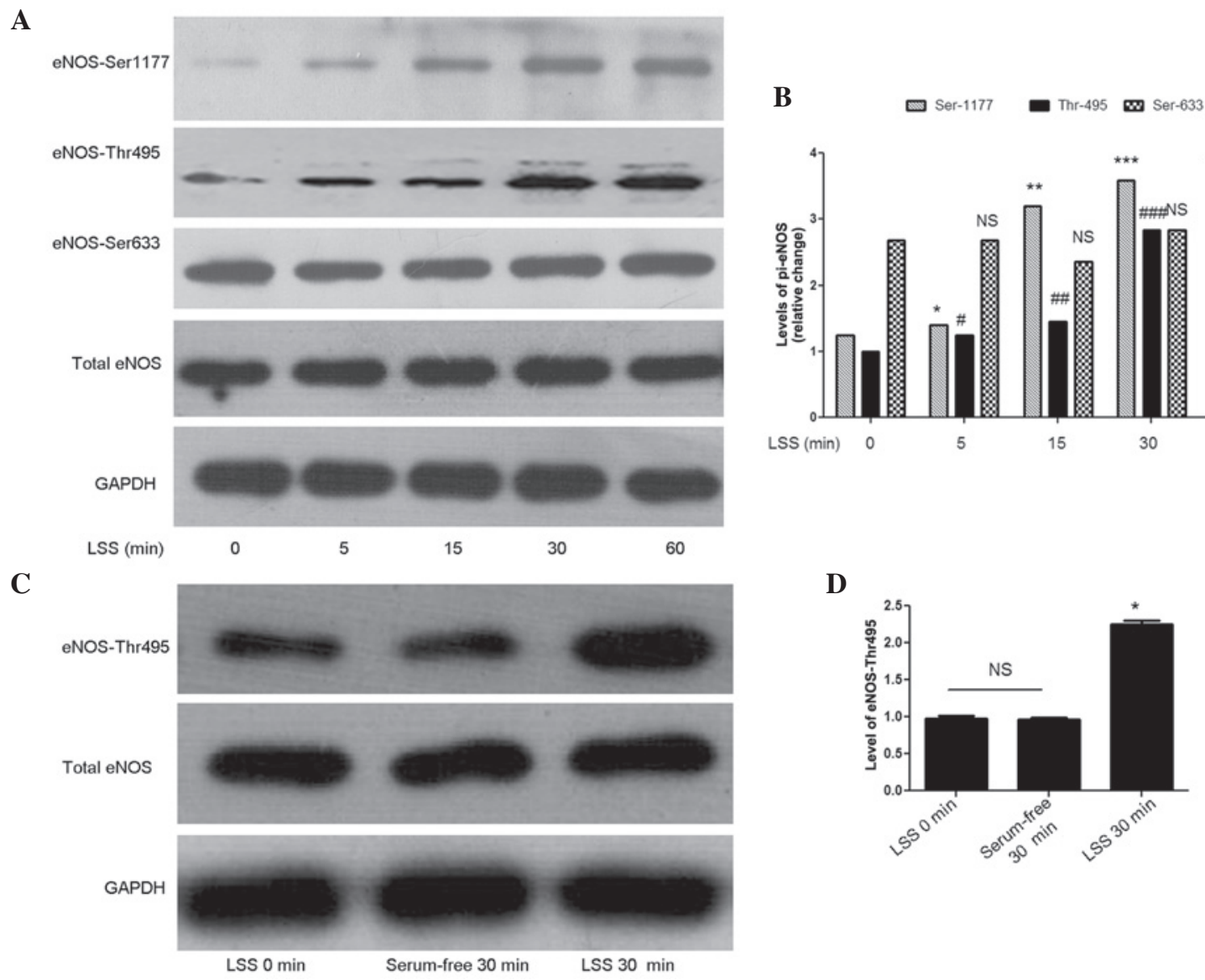

Figure 5. LSS modulates the phosphorylation of eNOS. (A) Representative immunoblots for phosphorylation of eNOS-Ser1177, eNOS-Ser633, eNOS-Thr495 and total eNOS in LSS-treated cells. The levels of proteins were normalized to GAPDH. (B) Densitometry analysis of immunoblots for phosphorylated eNOS-Ser1177, eNOS-Thr495 and eNOS-Ser633. " $\mathrm{P}<0.05$ vs. LSS $0 \mathrm{~min} ;{ }^{* * *} \mathrm{P}<0.05$ vs. LSS $5 \mathrm{~min} ;{ }^{* * * *} \mathrm{P}<0.05$ vs. LSS $15 \mathrm{~min} ;{ }^{*} \mathrm{P}<0.05$ vs. LSS 0 min; ${ }^{\# \#} \mathrm{P}<0.05$ vs. LSS 5 min; \#\#\# $\mathrm{P}<0.05$ vs. LSS $15 \mathrm{~min} ; \mathrm{n}=3$ /group. (C) Representative eNOS-Thr495, total eNOS and GAPDH in cells with either serum-free medium or LSS-treated for 30 min. (D) Densitometry analysis of eNOS-Thr495/eNOS. "P<0.05 vs. LSS 0 min; n=3/group. LSS, low shear stress; eNOS, endothelial nitric oxide synthase; NS, not significant.

FBS with serum-free medium may induce stress. To examine this possibility, eNOS-Thr495 activation in cells starved for $30 \mathrm{~min}$ and cells treated with LSS for 30 min were compared (Fig. 5C). As expected, the activation of eNOS-Thr495 was due to the effect of LSS and was not a result of serum-free stimulus.

ERK inhibition suppresses LSS-mediated phosphorylation of eNOS-Thr-495. With LSS, the activation of ERK reached a maximum level at $15 \mathrm{~min}$ (Fig. 6A). To investigate the hypothesis that ERK may serve as an upstream modulator of LSS-mediated eNOS, PD98059, an inhibitor of ERK, was used to treat the cells prior to the initiation of LSS for $30 \mathrm{~min}$. To ensure that an effective ERK inhibition was achieved, phospho-ERK expression was analyzed, which was $78 \%$ reduced in cells pre-incubated with PD98059 $(50 \mu \mathrm{mol} / \mathrm{l})$ compared with the control. Subsequently, the effect of LSS on eNOS-Thr495 was examined and the results demonstrated that ERK inhibition suppressed LSS-mediated activation of eNOS-Thr495 (Fig. 6E). Additionally, SOD activity was measured to analyze the antioxidant cellular defenses of ERK inhibition and the result revealed that PD98059 induced an increase in SOD activity, as compared with the control conditions (Fig. 6G). In conclusion, LSS-induced oxidative stress is regulated by the ERK/eNOS-Thr495 signaling pathway.
RSV blocks LSS-induced activation of ERK/eNOS. The preliminary results suggested the presence of a signaling pathway via LSS/ERK/eNOS. With the aim of investigating the role of RSV, phospho-ERK expression in the presence or absence of RSV in LSS-treated cells was evaluated. Compared with the control, RSV decreased ERK activation at LSS $15 \mathrm{~min}$ (Fig. 7A). As the maximum LSS-mediated stimulation of the phosphorylation of eNOS-Thr495 occurred at $30 \mathrm{~min}$ (Fig. 5A), this time-point was selected to examine the effect of RSV on eNOS and a decrease in eNOS-Thr495 levels was identified. In RSV-treated cells, eNOS Ser-633 was increased, while it was not altered following a short exposure to LSS. Unexpectedly, RSV deactivated LSS-phosphorylated eNOS-Ser1177 (Fig. 7C). LSS has been reported to increase cell permeability (26), which allows simple access for RSV to the cell, thus resulting in an altered activation of eNOS. Therefore, $\mathrm{LDH}$, which was released from the permeability-increased cells, was tested. The intracellular LDH assay demonstrated that RSV restored LSS-mediated increases in cell permeability (Fig. 7G). These results suggested that the antioxidant effect of RSV may partly be due to the suppression of ERK/eNOS-Thr495.

Acute exposure to LSS does not alter the expression of eNOS $m R N A$. qPCR was performed to investigate whether LSS 
A

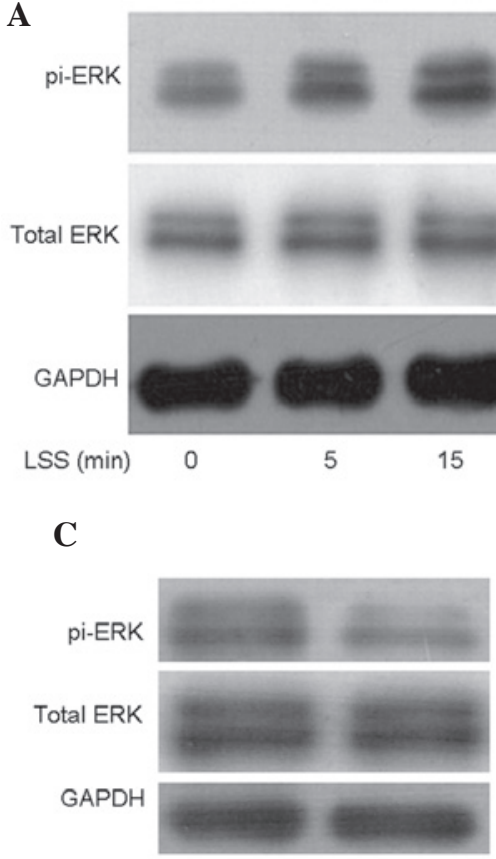

DMSO PD98059
D
B

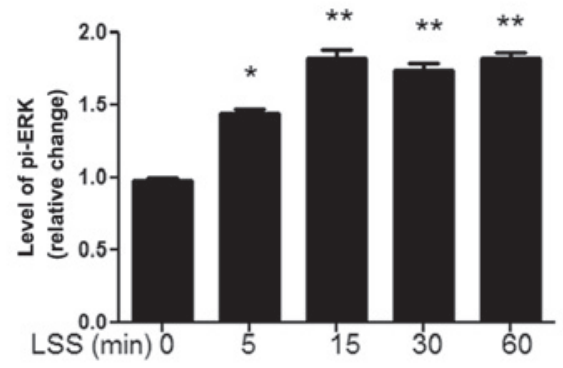

30

60
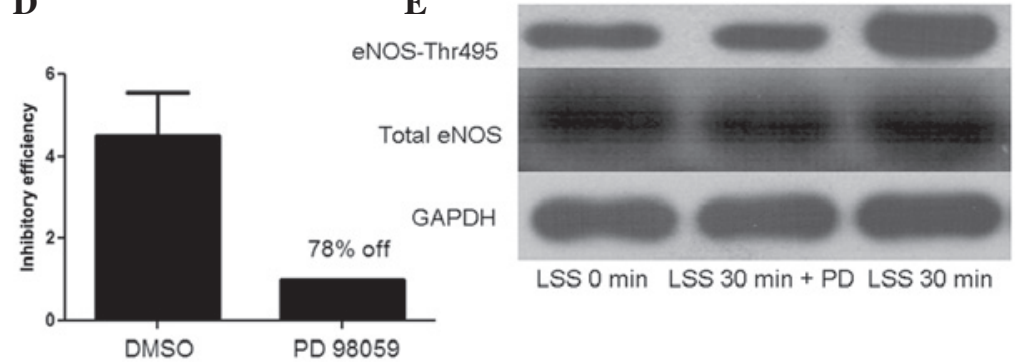

F

G
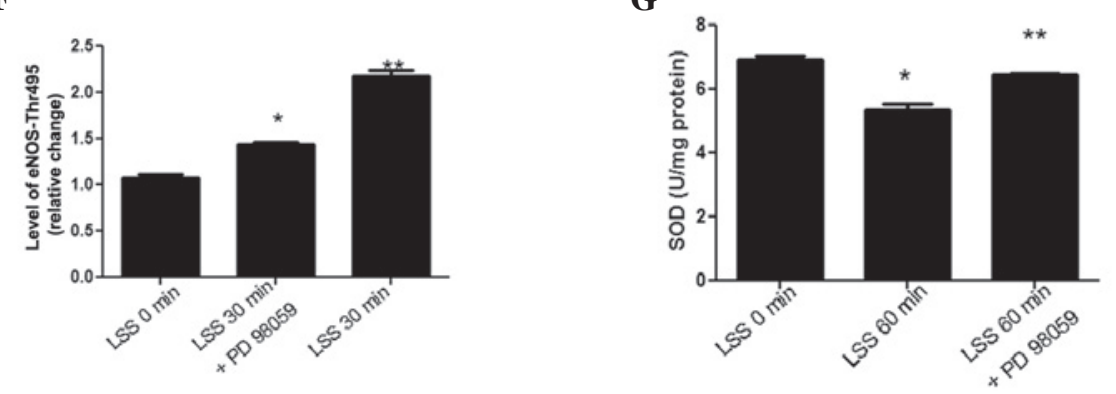

Figure 6. Inhibition of ERK blocks the LSS-induced activation of eNOS-Thr-495. (A) Representative immunoblots of phospho-ERK, ERK and GAPDH in cells with LSS treated for different duration. (B) Densitometric analysis of the expressed phospho-ERK. *P<0.05 vs. LSS 0 min and ** P<0.05 vs. LSS 5 min. (C) The effect of ERK inhibition on the expression of eNOS-Thr495, total eNOS and GAPDH in cells pretreated with PD98059 (50 $\mu$ mol/1), an ERK inhibitor, following the initiation of LSS for $30 \mathrm{~min}$. (D) Analysis of the inhibitory efficiency of PD98059; (E) quantitative analysis of Thr-495 eNOS and (F) densitometry analysis of eNOS-Thr495. ${ }^{*} \mathrm{P}<0.05$ vs. LSS $0 \mathrm{~min} ;{ }^{* *} \mathrm{P}<0.05$ vs. LSS $30 \mathrm{~min}+\mathrm{PD} 98059$. (G) Effect of ERK inhibition on intracellular SOD activity. ${ }^{*} \mathrm{P}<0.05$ vs. LSS $0 \mathrm{~min}$; ${ }^{\#} \mathrm{P}<0.05$ vs. LSS $60 \mathrm{~min}$; n=3/group. pi-ERK, phosphorylated extracellular signal-regulated kinase; LSS, low shear stress; eNOS, endothelial nitric oxide synthase; SOD, superoxide dismutase; DMSO, dimethyl sulfoxide.

contributed to the change in eNOS mRNA expression. No significant difference in eNOS mRNA levels was observed following LSS exposure for 0 and $60 \mathrm{~min}$ in the presence or absence of RSV (Fig. 8). This result suggested that LSS modulated eNOS in a post-transcriptional manner. Additionally, activated protein phosphorylation independent from altered gene expression is likely to have contributed to the modulation of eNOS in the short time flow experiment.

\section{Discussion}

In the present study, the antioxidant effect of RSV on LSS-treated cells was investigated and the possible mechanism that may be involved in the modulation of eNOS was examined. The results demonstrated that i) LSS evokes increased oxidative stress in ECs which may be due to the activation of ERK/eNOS-Thr495 and ii) RSV alleviates LSS-induced oxidative damage via suppressing the ERK/eNOS-Thr495 signaling pathway.
Increases in ROS levels have been well-defined as a mechanism for NO scavenging, leading to endothelial dysfunction (24). Multiple factors have been identified to enhance ROS formation in ECs, including mitochondrial dysfunction and eNOS uncoupling (25). By specific detection of mitochondrial superoxide by MitoSOX, the present study demonstrated that LSS augmented ROS generation. Oxidative stress causes cellular injury, and initiates apoptosis, which disrupts the integrity of the endothelium, increases endothelial permeability, and therefore leads to a preferred cellular deposition to lipids (26). Using TUNEL staining, apoptosis caused by LSS was identified, which indicated that LSS caused oxidative damage.

Three decades ago, Furchgott and Zawadzki (27) provided the first evidence that ECs produced a factor which caused vascular smooth muscle relaxation. Considering the observation was associated with NO, eNOS had since been intensely studied (28). Cheng et al (4) reported an association between the distribution of shear stress and differential activation of eNOS, which prompted us to investigate the effects of 
A

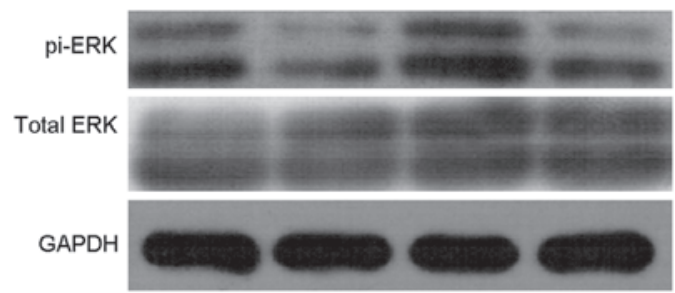

C

$\begin{array}{ccccc}\text { LSS (min) } & 0 & 0 & 15 & 15 \\ \text { RSV }(\mu \mathrm{mol} / \mathrm{l}) & 0 & 50 & 0 & 50\end{array}$

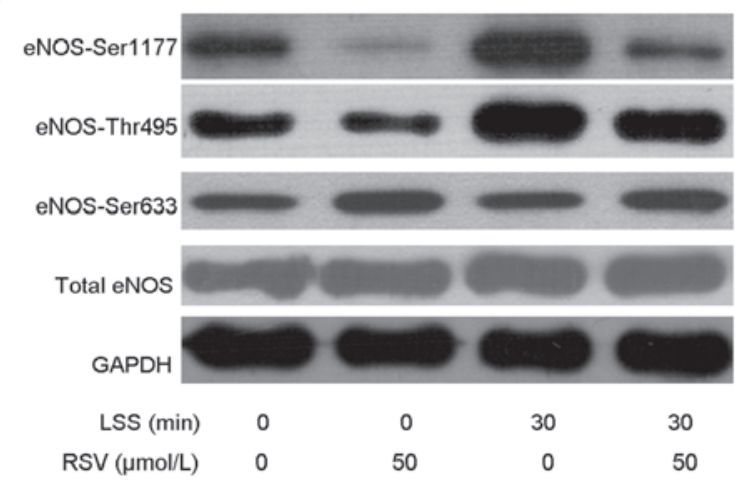

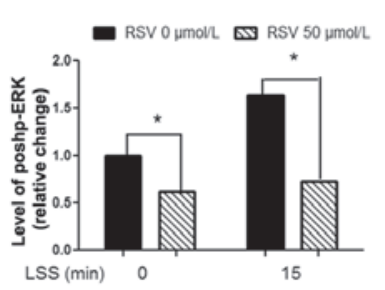

D

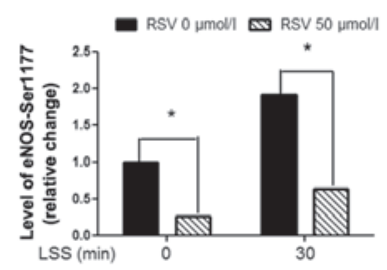

F

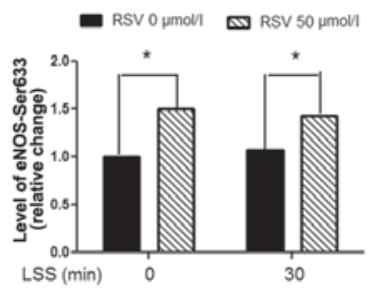

$\mathbf{E}$

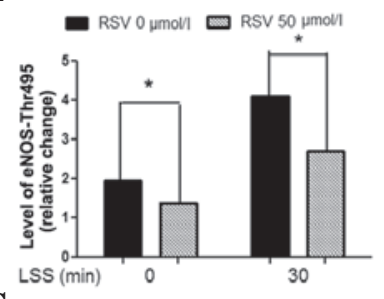

G

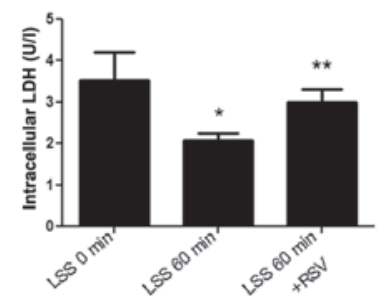

Figure 7. RSV suppresses the LSS/ERK/eNOS signaling pathway. (A) Representative immunoblots for phospho-ERK and ERK in cells exposed to LSS with or without RSV treatment and (B) analysis of phospho-ERK. (C) Representative blots for Ser1177, Thr-495, Ser-633 and total eNOS in RSV-treated cells. Densitometry analysis of immunoblots for the levels of (D) eNOS-Ser1177, (E) eNOS-Thr495 and (F) eNOS-Ser633. *P<0.05 vs. LSS 0 min without RSV; $\mathrm{n}=3$ /group. Cells subjected to LSS for different time periods were lysed to determine intracellular LDH. (G) LSS caused a decrease in intracellular LDH, which was restored by RSV treatment. ${ }^{* *} \mathrm{P}<0.05$ vs. LSS 60 min with no RSV treated; $\mathrm{n}=3$ /group. RSV, resveratrol; LSS, low shear stress; eNOS, endothelial nitric oxide synthase; ERK, extracellular signal-regulated kinase; LDH, lactate dehydrogenase.

LSS on eNOS multi-phosphorylation sites (4). Activation of Ser1177 or Ser633 promoted eNOS, while phosphorylation at Thr-495 had an inhibitory effect (7). Based on the above results and the evidence from the present study that LSS promoted ROS formation and decreased NO production, the phosphorylation at eNOS-Ser1177, Ser633 and Thr495 in ECs exposed to LSS was examined. The results demonstrated that the activation of eNOS-Ser633 remained unchanged and that Thr495 was elevated at $5 \mathrm{~min}$ and peaked at $30 \mathrm{~min}$ following the onset of flow. Unexpectedly, a short exposure to LSS evoked eNOS-Ser1177, which contradicted the original hypothesis that LSS inhibited eNOS-Ser1177 due to promoting the release of NO $(6,10)$. Greif et al (29) identified phosphatase protein phosphatase 2A (PP2A) as a key determinant of eNOS dephosphorylation at Ser1177 and Thr495 sites. Furthermore, an inhibitor of PP2A augmented overall levels of eNOS phosphorylation and a mutation at Thr495 deactivated Ser1177. In conclusion, it was proposed that PP2A is involved in LSS-activated eNOS and that short-term LSS activates Ser1177 in a Thr495-dependent manner; however, further study is required to fully elucidate this.

MAPK signaling is important in a wide range of cellular processes. ERK, one of the major factors in the MAPK family, responds to a variety of extracellular stresses (30). First, the effect of LSS on ERK was examined and it was observed that ERK was activated and peaked at $15 \mathrm{~min}$. To identify the upstream regulator of eNOS, PD98059 $(50 \mu \mathrm{mol} / \mathrm{ml})$ was used to pre-incubate cells for $30 \mathrm{~min}$ prior to the flow study.

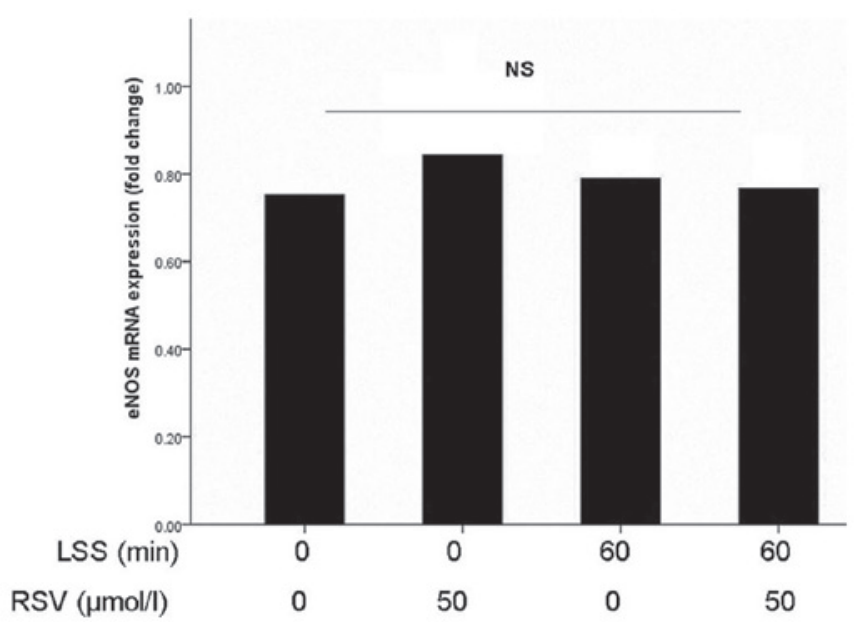

Figure 8. LSS modulates eNOS mRNA expression in a post-transcriptional manner. The levels of eNOS mRNA relative to GAPDH following exposure to LSS for 0 and $30 \mathrm{~min}$ did not exhibit any significant differences among the four groups; $n=4$ /group. LSS, low shear stress; eNOS, endothelial nitric oxide synthase; RSV, resveratrol; NS, not significant.

The results suggested that PD98059 suppressed LSS-induced activation of eNOS-Thr495 and restored SOD activity. These results corresponded with those obtained in other studies, including that by Huang et al (30), who identified that ERK affects eNOS under shear stress in a mouse model, and a study by Ford and Rush (25) also reported that activation of VEGF/ERK affects NO synthesis. 
RSV is a natural polyphenol that has been demonstrated to attenuate oxidative stress $(17,20,31)$. Substantial evidence suggested that the antioxidant effect of RSV is attributed to the activation of sirtuins, which are mainly located in the mitochondria (17). Several other studies have demonstrated that RSV may increase NO availability through the attenuation of ROS production (32). However, the mechanism by which RSV affects eNOS in LSS-treated cells is elusive. The present findings suggested that RSV attenuated oxidative stress and cell apoptosis, and affected ERK/eNOS signaling, suggesting that RSV improved the release of NO by blocking ERK/eNOS-Thr495. Furthermore, RSV was identified to regulate MAPK in several other studies $(30,33)$. Skrobuk et al (34) reported that an acute exposure to RSV inhibited AMPK activity, which may explain why RSV deactivated eNOS-Ser1177 in the present study (34). Additionally, the manner of RSV-regulated eNOS deactivation was in accordance with that mediated by PP2A (29). To eliminate the possibility that the antioxidant effect of RSV may be a result of increased cell permeability generated by LSS (11), intracellular LDH was measured. The results revealed that RSV restored the integrity of the cell membrane. Under static conditions, RSV affected the signaling of ERK/eNOS. However, the ROS and NO levels, as well the apoptotic rate of the cells, were independent of RSV under static conditions. RSV failed to affect oxidative damage in static cultured cells, suggesting that mechanisms other than eNOS may be associated with the modulation of oxidative stress.

In conclusion, it is proposed that i) LSS causes oxidative stress via ERK/eNOS-Thr495 and ii) RSV restores oxidative damage through suppressing ERK/eNOS-Thr495. Furthermore, PP2A may be a key regulator in LSS-affected eNOS and that LSS activates Ser1177 in a Thr495-dependent way, which are hypotheses that require further study.

There were certain limitations to the present study. Atherosclerosis is considered to be a chronic and complex process, and therefore, an extended exposure time in vivo should be applied. The present study suggested that LSS-induced oxidative damage was associated with ERK/eNOS-Thr495. PI3K/PKD/eNOS-Ser1177 and PKA/eNOS-Ser633 have been reported to be involved in long-term high shear stress $(35,36)$. High shear stress was also identified to stimulate nuclear export of histone deacetylase 5 and activate eNOS (37). However, these signaling pathways are characterized by large and complicated networks, and other key factors cannot be excluded, which requires further investigation.

\section{Acknowledgements}

This study was generously supported by a grant from the National Natural Science Foundation of China (no. 81270191).

\section{References}

1. Caro CG, Fitz-Gerald JM and Schroter RC: Arterial wall shear and distribution of early atheroma in man. Nature 223: 1159-1160, 1969.

2. VanderLaan PA, Reardon CA and Getz GS: Site specificity of atherosclerosis: site-selective responses to atherosclerotic modulators. Arterioscler Thromb Vasc Biol 24: 12-22, 2004.

3. Qin X, Tian J, Zhang P, Fan Y, Chen L, Guan Y, Fu Y, Zhu Y, Chien $S$ and Wang N: Laminar shear stress up-regulates the expression of stearoyl-CoA desaturase-1 in vascular endothelial cells. Cardiovasc Res 74: 506-514, 2007.
4. Cheng C, Tempel D, Oostlander A, Helderman F, Gijsen F, Wentzel J, van Haperen R, Haitsma DB, Serruys PW, van der Steen AF, de Crom $\mathrm{R}$ and Krams R: Rapamycin modulates the eNOS vs. shear stress relationship. Cardiovasc Res 78: 123-129, 2008.

5. Cheng C, van Haperen R, de Waard M, van Damme LC, Tempel D, Hanemaaijer L, van Cappellen GW, Bos J, Slager CJ, Duncker DJ, van der Steen AF, de Crom R and Krams R: Shear stress affects the intracellular distribution of eNOS: direct demonstration by a novel in vivo technique. Blood 106: 3691-3698, 2005.

6. Dimmeler S, Fleming I, Fisslthaler B, Hermann C, Busse R and Zeiher AM: Activation of nitric oxide synthase in endothelial cells by Akt-dependent phosphorylation. Nature 399: 601-605, 1999.

7. Sessa WC: eNOS at a glance. J Cell Sci 117: 2427-2429, 2004.

8. Le Brocq M, Leslie SJ, Milliken P and Megson IL: Endothelial dysfunction: from molecular mechanisms to measurement, clinical implications, and therapeutic opportunities. Antioxid Redox Signal 10: 1631-1674, 2008.

9. Atkins GB and Simon DI: Interplay between NF-kappaB and Kruppel-like factors in vascular inflammation and atherosclerosis: location, location, location. J Am Heart Assoc 2: e000290, 2013

10. Mount PF, Kemp BE and Power DA: Regulation of endothelial and myocardial NO synthesis by multi-site eNOS phosphorylation. J Mol Cell Cardiol 42: 271-279, 2007.

11. Cancel LM and Tarbell JM: The role of mitosis in LDL transport through cultured endothelial cell monolayers. Am J Physiol Heart Circ Physiol 300: H769-H776, 2011.

12. Yu W, Ying H, Tong F, Zhang C, Quan Y and Zhang Y: Protective effect of the silkworm protein $30 \mathrm{Kc} 6$ on human vascular endothelial cells damaged by oxidized low density lipoprotein (Ox-LDL). PLoS One 8: e68746, 2013.

13. Guo H, Chen Y, Liao L and Wu W: Resveratrol protects HUVECs from oxidized-LDL induced oxidative damage by autophagy upregulation via the AMPK/SIRT1 pathway. Cardiovasc Drugs Ther 27: 189-198, 2013.

14. Malinowska J, Oleszek W, Stochmal A and Olas B: The polyphenol-rich extracts from black chokeberry and grape seeds impair changes in the platelet adhesion and aggregation induced by a model of hyperhomocysteinemia. Eur J Nutr 52: 1049-1057, 2013.

15. Takizawa Y, Kosuge Y, Awaji H, Tamura E, Takai A, Yanai T, Yamamoto R, Kokame K, Miyata T, Nakata R and Inoue H: Up-regulation of endothelial nitric oxide synthase (eNOS), silent mating type information regulation 2 homologue 1 (SIRT1) and autophagy-related genes by repeated treatments with resveratrol in human umbilical vein endothelial cells. Br J Nutr 110: 2150-2155, 2013.

16. Quincozes-Santos A, Bobermin LD, Latini A, Wajner M, Souza DO, Goncalves CA and Gottfried C: Resveratrol protects C6 astrocyte cell line against hydrogen peroxide-induced oxidative stress through heme oxygenase 1. PLoS One 8: e64372, 2013.

17. Price NL, Gomes AP, Ling AJ, Duarte FV, Martin-Montalvo A, North BJ, Agarwal B, Ye L, Ramadori G, Teodoro JS, Hubbard BP, Varela AT, Davis JG, Varamini B, Hafner A, Moaddel R, Rolo AP, Coppari R, Palmeira CM, de Cabo R, Baur JA and Sinclair DA: SIRT1 is required for AMPK activation and the beneficial effects of resveratrol on mitochondrial function. Cell Metab 15: 675-690, 2012.

18. Rajapakse AG, Yepuri G, Carvas JM, Stein S, Matter CM, Scerri I, Ruffieux J, Montani JP, Ming XF and Yang Z: Hyperactive S6K1 mediates oxidative stress and endothelial dysfunction in aging: inhibition by resveratrol. PLoS One 6: e19237, 2011.

19. Kao CL, Chen LK, Chang YL, Yung MC, Hsu CC, Chen YC, Lo WL, Chen SJ, Ku HH and Hwang SJ: Resveratrol protects human endothelium from $\mathrm{H}(2) \mathrm{O}(2)$-induced oxidative stress and senescence via SirT1 activation. J Atheroscler Thromb 17: 970-979, 2010.

20. Arunachalam G, Yao H, Sundar IK, Caito S and Rahman I: SIRT1 regulates oxidant- and cigarette smoke-induced eNOS acetylation in endothelial cells: role of resveratrol. Biochem Biophys Res Commun 393: 66-72, 2010.

21. Becatti M, Taddei N, Cecchi C, Nassi N, Nassi PA and Fiorillo C: SIRT1 modulates MAPK pathways in ischemic-reperfused cardiomyocytes. Cell Mol Life Sci 69: 2245-2260, 2012.

22. Elíes J, Cuíñas A, García-Morales V, Orallo F and Campos-Toimil M: Trans-resveratrol simultaneously increases cytoplasmic $\mathrm{Ca}(2+)$ levels and nitric oxide release in human endothelial cells. Mol Nutr Food Res 8: 1237-1248, 2011. 
23. Min Z, Kang L, Lin L, Jinghua F, Junna S and Baolin L: Resveratrol restores lysophosphatidylcholine-induced loss of endothelium-dependent relaxation in rat aorta tissue coinciding with inhibition of extracellular-signal-regulated protein kinase activation. Phytother Res 12: 1762-1768, 2010.

24. Qiu X, Brown K, Hirschey MD, Verdin E and Chen D: Calorie restriction reduces oxidative stress by SIRT3-mediated SOD2 activation. Cell Metab 12: 662-667, 2010.

25. Ford RJ and Rush JW: Endothelium-dependent vasorelaxation to the AMPK activator AICAR is enhanced in aorta from hypertensive rats and is NO and EDCF dependent. Am J Physiol Heart Circ Physiol 300: H64-H75, 2011.

26. Conklin BS, Vito RP and Chen C: Effect of low shear stress on permeability and occludin expression in porcine artery endothelial cells. World J Surg 31: 733-743, 2007.

27. Furchgott RF and Zawadzki JV: The obligatory role of endothelial cells in the relaxation of arterial smooth muscle by acetylcholine. Nature 288: 373-376, 1980.

28. Palmer RM, Ferrige AG and Moncada S: Nitric oxide release accounts for the biological activity of endothelium-derived relaxing factor. Nature 327: 524-526, 1987.

29. Greif DM, Kou R and Michel T: Site-specific dephosphorylation of endothelial nitric oxide synthase by protein phosphatase $2 \mathrm{~A}$ : evidence for crosstalk between phosphorylation sites. Biochemistry 41: 15845-15853, 2002.

30. Huang A, Yang YM, Yan C, Kaley G, Hintze TH and Sun D Altered MAPK signaling in progressive deterioration of endothelial function in diabetic mice. Diabetes 61: 3181-3188, 2012.
31. Carrizzo A, Puca A, Damato A, Marino M, Franco E, Pompeo F, Traficante A, Civitillo F, Santini L, Trimarco V and Vecchione C: Resveratrol improves vascular function in patients with hypertension and dyslipidemia by modulating NO metabolism. Hypertension 62: 359-366, 2013.

32. Schmitt CA, Heiss EH and Dirsch VM: Effect of resveratrol on endothelial cell function: molecular mechanisms. Biofactors 36: 342-349, 2010.

33. Chan CM, Chang $\mathrm{HH}$, Wang VC, Huang $\mathrm{CL}$ and Hung $\mathrm{CF}$ : Inhibitory effects of resveratrol on PDGF-BB-induced retinal pigment epithelial cell migration via PDGFR $\beta$, PI3K/Akt and MAPK pathways. PLoS One 8: e56819, 2013.

34. Skrobuk P, von Kraemer S, Semenova MM, Zitting A and Koistinen HA: Acute exposure to resveratrol inhibits AMPK activity in human skeletal muscle cells. Diabetologia 55: 3051-3060, 2012.

35. Boo YC, Hwang J, Sykes M, Michell BJ, Kemp BE, Lum H and Jo H: Shear stress stimulates phosphorylation of eNOS at Ser(635) by a protein kinase A-dependent mechanism. Am J Physiol Heart Circ Physiol 283: H1819-H1828, 2002.

36. Boo YC, Sorescu G, Boyd N, Shiojima I, Walsh K, Du J and Jo H: Shear stress stimulates phosphorylation of endothelial nitric-oxide synthase at Ser1179 by Akt-independent mechanisms: role of protein kinase A. J Biol Chem 277: 3388-3396, 2002.

37. Wang W, Ha CH, Jhun BS, Wong C, Jain MK and Jin ZG: Fluid shear stress stimulates phosphorylation-dependent nuclear export of HDAC5 and mediates expression of KLF2 and eNOS. Blood 115: 2971-2979, 2010. 